\title{
Optically Nonlinear Polymeric Materials
}

\section{Development and Applications}

\author{
G.R. Möhlmann
}

Akzo Research Laboratories, Arnhem, The Netherlands

\begin{abstract}
Optically nonlinear materials exhibit changes of their refractive index if placed in electric, magnetic or optic fields. Such materials can be used as active media in devices and integrated optics to manipulate light.
\end{abstract}

Electro-optic devices provide basic functions such as: phase or intensity modulation, optical switching, frequency doubling and mixing, etc. for optical communication, processing, computing and storage systems. For example, integrated electro-optic phase modulators can be used for signal processing. Furthermore, if the phase modulator is integrated in one arm of a Mach-Zehnder interferometer, intensity switching in addition to phase modulation can be obtained. On bringing two or more parallel channel waveguides within a few microns of each other, electro-optically induced coupling can give rise to the optical space switching of signals. Finally thermo-optically induced phase changes in liquid crystalline versions of polymers can be used for optical storage devices.

All materials when examined at the atomic or molecular level reveal an assembly of positively and negatively charged particles such as ions and electrons, which can be displaced by an external electric field. The corresponding separation of the centres of gravity of the positive and negative charges is called induced electrical polarization. For weak external fields, the induced polarization is nearly proportional to the field strength. For strong fields, however, the induced polarization may exhibit noticeable nonlinear contributions that are at the root of optically nonlinear phenomena.

In principle, all materials show optically nonlinear effects if placed in suffi-

Dr. Gustaaf Möhlmann has been with the Applied Physics Group, Corporate Research Dept., Akzo Research Laboratories, Verlperweg 76, P.O. Box 9300,6800 SB Arnhem, The Netherlands, for 12 years where he is responsible for the development of polymeric optically nonlinear devices. Prior to this he studied theoretical organic chemistry and experimental physics at the University of Leiden. ciently strong fields, although at times, particular symmetry requirements may need to be fulfilled. The expression "optically nonlinear materials" is generally used for materials showing significant induced nonlinear polarization at small or moderate applied voltages.

It is believed that organic polymers will become attractive optically nonlinear materials, finding their place in applications. Some developments and results will be discussed.

\section{Nonlinear Polarization}

The induced electrical polarization $(P)$ can be expressed as a function of linear and nonlinear terms of the electric field strength $E$, by [1]:

$P(E)=\varepsilon_{0} \cdot E\left[\chi^{(1)}+\chi^{(2)} \cdot E+\chi^{(3)} \cdot E^{2}+\ldots\right]$ in which $\varepsilon_{\mathrm{o}}$ is the dielectric permittivity in vacuo and $\chi^{(\mathrm{n})}$ the $\mathrm{n}$-th order dielectric susceptibility. $\chi^{(n)}$ and $E^{n}$ are in reality tensor elements but are here for simplicity written without further indices. The term $\chi^{(1)}$ is connected to the refractive index $n$ via the relation $\chi^{(1)}=$ $n^{2}-1$. The other terms, $\chi^{(2)} \cdot E, \chi^{(3)} \cdot E^{2}$, etc., have the same dimension as $\chi^{(1)}$ and can thus be considered as field strength dependent changes of the refractive index. However, whereas the odd susceptibility terms are always non-zero, if the even terms do not equal zero there can be no centrosymmetry (inversion centre) in the system.

Only a small fraction of all materials are spontaneously non-centrosymmetric and show second order optically nonlinear effects such as: (linear) electro-optic (or Pockels) effects, frequency doubling, frequency mixing, etc. Third order effects include quadratic electrooptic effects, frequency tripling, stimulated scattering and other opto-opto effects. Higher order nonlinearities usually have very small coefficients and are rarely of practical importance; they will not be discussed.

\section{Optically Nonlinear Materials}

The best-known second order nonlinear materials are the inorganic single crystals like: quartz, potassium-dihydrogen-phosphate (KDP), lithium niobate, lithium iodate, barium titanate, etc. in which the displaceable charges are positive and negative ions. Since ions have relatively large masses, and thus cannot easily respond to very rapidly oscillating (e.g. optical) fields, the coefficients for DC or low frequency induced electro-optic effects are considerably larger than those for frequency doubling (rapidly oscillating) optical fields, where electronic displacements are believed to be the main contributors. lons are tightly bound to their positions in a crystal leading to small amplitudes of the nonlinear effects. Nevertheless, inorganic single crystals are often used: KDP in Pockels cells and as frequency doublers in lasers, lithium niobate in guided-wave phase modulators, etc. .

Materials used for third order nonlinear effects are quartz, glass, lead and semi-conductor doped glasses, etc. Applications are in the field of opto-opto directional mode coupling, dynamic grating formation, etc. Their use is often hampered by the rather complex crystal growing and processing steps and alternative materials which also show improved properties with respect to the magnitude of the nonlinear effect are being sought. Electrons in conjugated electron systems seem to be ideal because they have displaceable charges of small mass with long range mobility. Organic molecules containing conjugated $\pi$-electron systems should be good materials and it has indeed been found that molecules such as p-nitro-aniline (p-NA) show important nonlinear optical effects. To simplify processing it is preferable to have these materials available in the form of polymers so as to make use of their formability combined with good compatibility with many materials.

\section{Organic Nonlinear Materials}

There is an important difference between the basic building blocks of inorganic and organic materials. In the former the composition of single ions is not the same as that of the bulk material whereas in the latter the molecules often have the same composition as the bulk. Consequently, the nonlinear pro- 
perties of organic materials are maintained down to the molecular level where they can still be characterized and optimized. In particular, the molecular analogue of the macroscopic induced polarization $P$, is the induced dipole moment $\mu$, and can be expressed as:

$$
\mu=\alpha E+\beta E^{2}+\gamma E^{3}+\ldots
$$

in which $\alpha$ is the (linear) polarizability, $\beta$ the hyperpolarizability, $\gamma$ the second hyperpolarizability, etc.

As in the macroscopic case, if molecular second order nonlinear effects ( $\beta$ not equal to zero) are desired then centrosymmetry is not allowed in the molecular structure. An attractive feature of organics is that non-centrosymmetric molecules can always be sythesised via asymmetric substitution. For instance, the centrosymmetric molecule stilbene (2nd order inactive) can be replaced by the nonlinear molecule 4-dimethylamino-4'-nitrostilbene (DANS) which possesses large nonlinear activity (see Fig.1).

The relation between molecular and macroscopic quantities in the case of second order nonlinear effects can be expressed as:

$$
\chi^{(2)}=(N N) \beta F<\cos ^{3} \varphi>
$$

in which $N N$ is the density of the nonlinear molecules, $F$ is a local field correction factor, and $\left\langle\cos ^{3} \varphi\right\rangle$ is the polar order parameter. For isotropic (centrosymmetric) systems, $\left\langle\cos ^{3} \varphi\right\rangle=0$ and no bulk second order nonlinear effect is present. Asymmetrically substituted (and thus optically nonlinear) molecules always possess a permanent dipole moment so they tend to align themselves in opposite directions (centrosymmetric) to each other in the bulk, resulting in inactive material. By adding proper groups (e.g. methyl) to a molecule to lower the symmetry further non-centrosymmetric crystallization can sometimes be obtained le.g. 2-methyl-4-nitro-aniline); in this case $\left\langle\cos ^{3} \varphi\right\rangle$ is not equal to zero.

In a polymer, the centrosymmetry that is always present after synthesis or deposition can be removed by aligning the permanent dipoles with an external electric field. On heating the polymer to a temperature above the glass transition temperature $T_{g}$, it reaches a flexible, rubbery state where movement of polymer segments, and consequently alignment of the nonlinear groupś, takes place. On cooling to below $T_{g}$ while maintaining the field, the induced polar order $\left(\left\langle\cos ^{3} \varphi\right\rangle\right)$ can be frozen in to give a second order, optically nonlinear polymeric material.

Organic nonlinear materials can be produced in different forms:

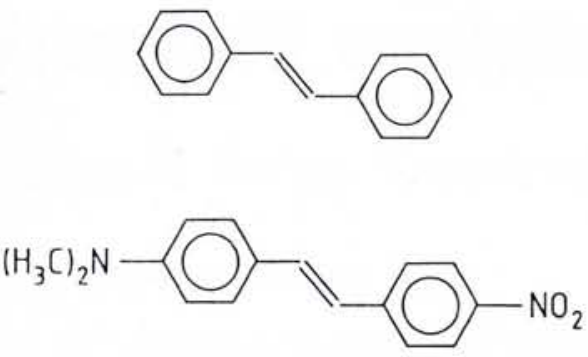

Fig. 1 - Structure of the molecules stilbene (upper) and DANS (lower).

Single crystals. Only a small fraction of the single crystals are non-centrosymmetric. Growth and processing is not easy and the most attractive application may be frequency doubling.

Langmuir-Blodgett (LB) films. Optically nonlinear materials may be produced via successive deposition of monolayers by the LB-technique while maintaining non-centrosymmetry. However, thus far it has not been possible to demonstrate the deposition of the several hundreds of layers needed for waveguides.

Solid solutions of nonlinear molecules in polymer matrices. A disadvantage here is the limited concentration of active molecules that can be introduced into the system owing to the tendency for segregation and subsequent centrosymmetric crystallization.

Main chain polymers in which the active groups are incorporated into the polymer back-bone permit an increase in the concentration of such groups without segregation. However, during the poling process, large segments of the polymer back-bone must be reoriented which is very difficult owing to the high apparent viscosity.

Side chain polymers with the active molecules attached to the polymer back-bone via a flexible spacer. This approach allows a high concentration of the incorporated nonlinear molecules combined with reduced relaxation after poling owing to one-sided anchoring of the molecules. Electric field poling is rather easy because only the relatively small active side groups need be reoriented.

\section{Side Chain Polymers}

Side chain polymers seem to be the most attractive organic nonlinear materials because they offer the best compromise between nonlinear optical properties and processing characteristics. In addition, organic synthetic chemistry offers the potential for independent optimisation (tailoring) of molecular and polymer properties. Some advantages of side chain polymers are:
- high concentration of nonlinear groups leading to large nonlinear bulk coefficients and effects;

- fast nonlinear response as electrons are the displaceable charges;

- low dielectric constant which means that high-speed electronic drive circuitry can be adopted;

- easy deposition from solution via spin coating, dipping, doctor blading, etc., and processing via etching, sputtering, etc.:

- deposition of large areas with selected thicknesses $(0.1-100 \mu \mathrm{m})$;

- compatibility with a variety of substrates (glass, plastics, metals, silicon, III-V semiconductors, etc.);

- low temperature deposition and processing permit monolithic integration with semiconductor IC's and opto-electronics;

- optical monomode waveguide formation via etching, etc. :

- 3-D architectures via successive layer deposition and processing;

- tailoring of molecular and polymeric properties ( $T_{g^{\prime}}$ absorption maximum, hardness, etc.) via organic synthesis.

Nonlinear polymers with a selected $T_{\mathrm{g}}\left(60^{\circ} \mathrm{C}<T_{\mathrm{g}}<140^{\circ} \mathrm{C}\right)$ have been produced on a kilogram scale by Akzo. The deposition of thin film multilayers has also been demonstrated. Electric field poling above the $T$, with field strengths on the order of $150 \mathrm{~V} / \mu \mathrm{m}$ resulted in optically nonlinear materials with large electro-optic coefficients.

\section{Multilayer Structures}

Multilayer structures that permit electric field poling and subsequent shaping must be realised in order to produce optically nonlinear devices. These multilayers generally comprise a substrate, bottom electrode, lower optical buffer (cladding), active core, upper buffer and top electrode. The active core layer must possess a higher refractive index than the buffer layers in order to ensure optical penetration into the core, although depending on the difference between the indices of the core and cladding, the light may or may not be entirely confined to the core. The role of the buffers is to separate the light beam geometrically from the electrodes thus avoiding unwanted absorption and scattering. The exceptions are the so-called "free space" devices such as tunable Fabry-Perot etalons where cladding is not required since light propagates out of the plane of the multilayer. A schematic representation of a multilayer structure, together with the intensity distribution of the propagating light beam in the slab-like waveguide core, is given in Fig. 2. 


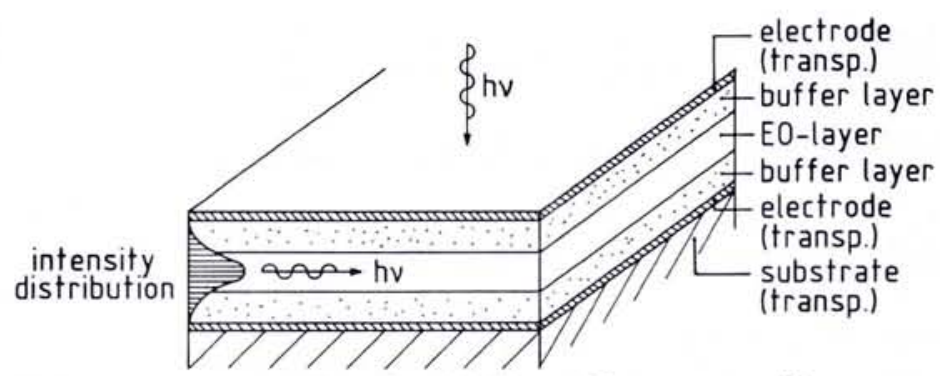

Fig. 2 - Schematic representation of a slab-like multilayer waveguide structure.

The electrodes can be applied by evaporation or sputtering and the polymer layers (buffer, core) by spin coating, dipping, etc. The electrode thickness is several tens of nanometers, while that of the polymer layers is of the order of microns. Monomode channel waveguides can be defined in the multilayer using reactive ion etching.

\section{Nonlinear Devices}

In order to apply and evaluate our optically nonlinear polymers, cooperative research efforts have been started with partners active in integrated optics and related technologies. Within the European Community supported programme "Research on Advanced Communication in Europe" (RACE Project R1019, "Polymeric Optical Switches"), researchers based at the $\mathrm{Dr}$. Neher Laboratories of the Dutch PTT, Leidschendam, and at Barr and Stroud, Glasgow, have produced a monomode channel waveguide phase modulator based on an Akzo polymer. The electroabout $130^{\circ} \mathrm{C}$ with a field strength of $156 \mathrm{~V} / \mu \mathrm{m}$. The electro-optic coefficient, $r_{33}$, which is related to $\chi^{(2)}$ via $\chi_{222}^{(2)}=$ $r_{33} n^{4} / 2$ was found to be equal to 28 $\mathrm{pm} / \mathrm{V}$ for $1.3 \mu \mathrm{m}$ propagating radiation; this $r_{33}$-values is among the highest reported [2, 3]. The $r_{33}$-value for lithium niobate is equal to $32 \mathrm{pm} / \mathrm{V}$ so a polymer with about the same nonlinear effect as for inorganic materials has been realised. optic effect was induced by poling at
The Laboratoires de Marcoussis, another partner in the RACE project, have built a polymeric integrated MachZehnder interferometer that exhibits an intensity modulation depth of about $15 \mathrm{~dB}$. This type of device can, if monolithically integrated with emitters and receivers, be used to tune and modulate laser output before it is injected into a telephone network, and to assist in detecting the correct signals at the receiving end.

At IMEC in Ghent (also a partner), polymeric tunable Fabry-Perot (free space) modulators have been condevices may form the basis of large area spatial light modulators for parallel optical image processing. In addition, under a separate bilateral Akzo/IMEC cooperation, the monolithic integration of an infrared diode laser with a polymeric multilayer waveguide has been demonstrated.

\section{REFERENCES}

[1] Nonlinear Optical Properties of Organic and Polymeric Materials, Ed. D.J. Williams, ACS Symposium Series 233 (American Chemical Society, Washington, D.C.) 1983. [2] Möhlmann G.R. et al., Proc. Topical Meeting on Nonlinear Guided Wave Phenomena: Physics and Applications IOSA, Washington, D.C.) 1989.

[3] Möhlmann G.R. et al., Recent Developments in Optically Nonlinear Polymers and Related Electro-optic Devices, Nonlinear Optical Properties of Organic Materials, ed. G. Khanarian, Proc. SPIE Conf., San Diego, 1989. structed for materials evaluation. Such

\section{Hubble Aloft}

The Hubble Space Telescope is a joint NASA/ESA project with a $15 \%$ participation from ESA. The first cycle of the so-called "General Observer Proposals" netted requests for 11000 hours of observing time using the telescope's five instruments. With only 1200 hours available and an average allocated time of 10 hours, an international panel selected just 162 proposals, $20 \%$ of which came from ESA Member States. The problem for astronomers is that the $20 \%$ allocation for Europe does not reflect the fact that its community is larger than the US's.

The Space Telescope Science Institute in Baltimore, USA will manage allocation and scheduling. The latter is a nightmare owing to numerous operational constraints including reflected sunlight, the earth's shadow, radiation from Van Allen belts, etc. Europe's centre for coordinating observations will be at the headquarters of the ESO in Garching, FRG.

While astronomers can spend up to a few weeks at the Institute in Baltimore, they can also send in proposals. Providing these are accepted, they will be sent a magnetic tape when the observations are completed. They will have one year to write up their work before the data falls into the public domain.

Now that the HST is finally aloft, just 44 years after the first serious study of the benefits of a special observatory in space, nearly seven years after its first expected launch date, we are awaiting reports of the first images as we go to press.

The telescope is scheduled to remain on station for 15 years with in-orbit repairs and replacement, circulating the earth every 95 minutes during which time it is can see a particular area for $\mathbf{4 5}$ minutes. Weighing 11 tonnes, it is the largest satellite ever to be lifted into space by the shuttle launcher. It will allow us to look back some seven times further in distance using cameras and detectors whose resolving power is a factor 10 better than for to-day's ground-based telescopes.

Europe was responsible for one of the cameras - the Faint Object Camera that counts individual photons in the UV, undisturbed by the earth's atmosphere, using exposures that will sometimes last up to 10 hours. Experts anticipate that some of the most exciting discoveries will not have been predicted so everyone is keeping their fingers crossed for a successful commissioning phase, lasting seven months, before Hubble starts to observe in earnest at the rate of about 20 images daily.

\section{EPS-ACAPPI Workshop (EIW-7), Leningrad, USSR}

The EPS Action Committee for Applied Physics and Physics in Industry is organizing a Europhysics Industrial Workshop titled "Semiconductor Surface Science and Engineering" that will take place in Leningrad on 19-22 June 1990.

The workshop is an "historic" first for the Society since representatives from eastern and western industries, institutes and universities will meet informally with Russian colleagues pre-eminent in the field to explore ways in which academically orientated research can assist industrial development.

As is usually the case for industrial workshops, the programme is not too densely packed to allow time for discussion. There will be seven sessions with a total of about 30 presentations, 12 of which will be made by experts from Russia and the remainder by a wellbalanced mixture of scientists from eastern and western Europe.

The organisers have been able to obtain funds to provide hotel accommodation for participants from outside Russia.

\section{Meanwhile, Back on Earth...}

The overall excess demand for Hubble (almost a factor 10 ) is perhaps not so surprising because the best ground-based telescopes must routinely cater for factors approaching four. The latter clearly remain advances such as active optics, that permits compensation for atmospheric disturbances, and arrays of telescopes. The decision on where to site one such European array comprising four separate telescopes with mirrors eight metres in diameter will be announced shortly. sought after, especially in view of technical 